\title{
Comparative Pitching Biomechanics Among Adolescent Baseball Athletes: Are There Fundamental Differences Between Pitchers and Non-pitchers?
}

\author{
Garrett Bullock, PT, DPT ${ }^{1}$, Tessa C Hulburt, MS², Gary Collins, Bsc (Hons), PhD, Brian R Waterman, MD², Kristen F \\ Nicholson, $\mathrm{PhD}^{2}$ \\ 1 Department of Orthopaedic Surgery, Wake Forest School of Medicine; Centre for Sport, Exercise and Osteoarthritis Research Versus Arthritis, \\ University of Oxford; Nuffield Department of Orthopaedics, Rheumatology, and Musculoskeletal Sciences, University of Oxford, ${ }^{2}$ Department of \\ Orthopaedic Surgery, Wake Forest School of Medicine, ${ }^{3}$ Centre for Statistics in Medicine, Nuffield Department of Orthopaedics, Rheumatology, and \\ Musculoskeletal Sciences, University of Oxford; Oxford University Hospitals NHS Foundation Trust \\ Keywords: pitch velocity, ground reaction force, trunk rotation velocity, adolescent, baseball \\ https://doi.org/10.26603/001c.21495
}

\section{International Journal of Sports Physical Therapy}

Vol. 16, Issue 2, 2021

\section{Background}

Approximately $25 \%$ of youth baseball players pitch, with most young athletes predominately playing multiple positions. While some youth baseball players may primarily pitch, other players may only pitch on occasion, potentially creating a pitching skill level discrepancy. Understanding potential kinematic and kinetic differences between pitching and non-pitching baseball players can inform injury risk reduction strategies for amateur athletes.

\section{Purpose/Hypothesis}

To analyze differences in pitching biomechanics for fastballs, breaking balls, and change-ups in adolescent youth baseball players that identify as pitchers and non-pitchers.

\section{Study Design}

Retrospective cross-sectional study

\section{Methods}

Baseball players were designated as pitchers or non-pitchers, who then threw fastballs (FB), breaking balls (BB), and change-ups $(\mathrm{CH})$ during a biomechanical assessment. T-tests, Mann-Whitney U tests, and ANOVAs with Bonferroni correction, and effect sizes (ES) were performed.

\section{Results}

Sixty baseball players (pitchers = 40; non-pitchers = 20; Age: 15.0 (1.1); Left-handed: 15\%; Height 1.77 (0.09) m; Weight: 70.0 (12.5) kg) threw 495 pitches (FB: 177, BB: 155, $\mathrm{CH}$ : 163) for analysis. Pitchers threw $2 \mathrm{~m} / \mathrm{s}$ faster and produced greater trunk rotation velocity (ES: 0.71 (95\% CI: 0.39, 1.30, p<0.0001) than non-pitchers. Furthermore, pitchers demonstrated greater ground reaction force for FB compared to $\mathrm{CH}$ (ES: 0.48 (95\% CI: $0.01,0.94), \mathrm{p}<0.0001)$. No other biomechanical differences were observed between pitchers and non-pitchers or between pitch types.

\footnotetext{
a Corresponding Author: Garrett S. Bullock PT, DPT

Nuffield Department of Orthopaedics, Rheumatology, and Musculoskeletal Sciences University of Oxford

B4495

Oxford, United Kingdom

OX3 7LD

(865) 227-374

garrett.bullock@wolfson.ox.ac.uk
} 


\section{Conclusion}

Despite throwing at greater velocity for all pitch types, baseball players that identify primarily as pitchers had overall similar kinematics and kinetics in comparison to baseball players that primarily identify as non-pitchers. Self-identified pitching baseball athletes have improved force transfer strategies for ball propulsion, utilizing different force production and attenuation strategies across different pitch types when compared to non-pitchers. Coaches should consider that novice pitchers may potentially have dissimilar trunk and ground reaction strategies in comparison to primary pitchers when designing appropriate pitch loading and recovery strategies.

\section{Level of Evidence}

\section{INTRODUCTION}

Youth baseball injuries remain a significant public health concern. ${ }^{1-4}$ There are over 15.6 million baseball participants in the United States, ${ }^{5}$ with 3 million playing Little League $^{6}$ and almost 500,000 participating at the high school level. ${ }^{7}$ Up to $50 \%$ of youth baseball pitchers report arm pain during pitching, with a $52 \%$ and $86 \%$ increased risk of pain among pitchers that throw a curveball or slider, respectively. ${ }^{8}$ Furthermore, approximately $25 \%$ of adolescent baseball players pitch, ${ }^{9}$ with the majority of young athletes predominately playing multiple positions. ${ }^{10}$ While some adolescent baseball players may primarily pitch, other players may only pitch on occasion, potentially creating a pitching skill level discrepancy.

Pitching a baseball is a series of complex, multifaceted movements that produce high forces throughout the entire kinetic chain. ${ }^{11}$ In order to handle these high forces, pitchers must attempt to minimize forces to the shoulder and elbow through proper pitching mechanics. ${ }^{11,12}$ For example, two pitchers may pitch at the same velocity, but the one pitcher may generate greater muscle and joint forces, producing decreased force directed towards ball propulsion. ${ }^{13,14}$ Decreasing pitching upper extremity forces has been associated with overall pitching skill, with less skilled pitchers potentially resulting in increased injury risk. ${ }^{15}$ On the other hand, highly skilled pitchers generate increased pitch force and velocity but are better protected from these potentially adverse effects due to more of these high forces directed to ball propulsion. ${ }^{11}$ Understanding how pitching kinematics and kinetics differ between baseball players that primarily pitch or only pitch on occasion can assist clinicians and coaches in determining injury risk reduction strategies across a broader range of adolescent baseball athletes.

Different pitch types and/or deliveries are an essential component of pitching success. The ability to produce similar pitching mechanics between different pitch types delays pitch type recognition by the hitter, improving chances of a successful pitch. ${ }^{16}$ While producing a similar pitching motion between pitch types is recommended, ${ }^{16}$ discrepancies in kinematics and kinetics have been observed at the youth level. ${ }^{16-18}$ Differences in fastball and curveball kinematics and kinetics have been observed at the wrist and hand; these differences are likely not clinically significant because of the limited wrist and hand injury prevalence in baseball. ${ }^{19-21}$ However, fastballs produce greater kinetic forces at the shoulder and elbow in comparison to breaking balls and change-ups, ${ }^{16-18}$ which can potentially alter injury risk. ${ }^{22}$ Further, differences in pitching forces have been observed for different pitch types between different skill levels, with higher skill levels producing greater force generation for ball propulsion compared to upper extremity forces. ${ }^{15}$

Currently, it is not understood if adolescent baseball players who identify as pitchers or non-pitchers demonstrate different pitching kinematics or kinetics. Given the increased risk and severity of injury among pitching athletes, ${ }^{8,10}$ understanding potential differences in pitching kinematics and kinetic strategies can inform clinicians and coaches on potential injury risk reduction strategies in nonpitchers who are asked to pitch in a game. Therefore, the purpose of this study was to analyze differences in pitching biomechanics for fastballs, breaking balls, and change-ups among adolescent baseball players that identify as pitchers and non-pitchers. The authors hypothesized that those identifying primarily as pitchers would produce different kinematics and kinetics in comparison to adolescent baseball players that identify as non-pitchers.

\section{METHODS}

\section{STUDY DESIGN}

Prior to verbal and written consent and participation, all participants and their parents or guardians were informed of the risk and benefits of study participation. This study was approved by the Wake Forest School of Medicine Institutional Review Board. Baseball players from regional high schools and baseball academies participated in a pitching evaluation at the Wake Forest Pitching Laboratory. Inclusion criteria consisted of adolescent baseball players from all competition levels and between the ages 12 and 18. Participants were able to participate in all standard training, practices, and competitions at initial testing, and they threw at least two different pitch types (fastball, breaking ball, or change-up) based on prior pitching experience. Data were excluded if participants reported pain during any testing, had undergone surgery in the past twelve months, or were not participating in baseball-related training, practices, or games at the time of evaluation. Baseball players were then designated, through consensus between participants, parents, and coaches as those that were primarily pitchers and those that identified as non-pitchers (primarily played other positions such as catcher or short stop) and 
Table 1: Participant characteristics

\begin{tabular}{|c|c|c|c|}
\hline & $\begin{array}{l}\text { All participants } \\
\quad(n=60)\end{array}$ & $\begin{array}{l}\text { Pitchers } \\
(n=40)\end{array}$ & $\begin{array}{c}\text { Non-Pitchers } \\
(n=20)\end{array}$ \\
\hline Age & $15.0(1.1)$ & $15.1(1.2)$ & $14.9(1.0)$ \\
\hline $\begin{array}{c}\frac{\text { Throwing Hand }}{\text { Left }} \\
\text { Right }\end{array}$ & $\begin{array}{c}9[15 \%] \\
51[85 \%]\end{array}$ & $\begin{array}{c}8[20 \%] \\
32[80 \%]\end{array}$ & $\begin{array}{c}1[5 \%] \\
19[95 \%]\end{array}$ \\
\hline Height (m) & $1.77(0.09)$ & $1.79(0.08)$ & $1.73(0.08)$ \\
\hline Weight (kg) & $70.0(12.5)$ & $70.5(10.8)$ & 68.9 (15.7) \\
\hline $\begin{array}{c}\text { Number of Pitches } \\
\text { Fastball } \\
\text { Curveball } \\
\text { Changeup }\end{array}$ & $\begin{array}{l}177[36 \%] \\
155[31 \%] \\
163[33 \%]\end{array}$ & $\begin{array}{l}116[34 \%] \\
110[32 \%] \\
116[34 \%)]\end{array}$ & $\begin{array}{l}61[40 \%] \\
45[29 \%] \\
47[31 \%]\end{array}$ \\
\hline
\end{tabular}

Means and standard deviations are reported as mean (SD), [ ] indicates percentage of the total number of observations.

who may pitch only on occasion.

\section{BIOMECHANICAL ANALYSIS}

Kinematic three dimensional (3D) motion data were collected using the 40 reflective marker set required for PitchTrak (Motion Analysis Corporation; Santa Rosa, California) with a sixteen-camera motion analysis system (Motion Analysis Corporation; Santa Rosa, California). Motion data were collected at $250 \mathrm{~Hz}$. Ground reaction forces (GRF) were collected with three multi-component force plates (AMTI; Watertown, Massachusetts) embedded in the Perfect Mound (Porta-Pro Mounds Inc; Sauget, Illinois). One plate was positioned under the pitching rubber with the front edge six inches in front of the rubber. The other two plates were angled at $4.8^{\circ}$ and covered the landing zone. Each plate was covered with artificial turf to match the rest of the mound. Force plate data were collected at $1000 \mathrm{~Hz}$. Pitchers threw from the Perfect Mound and were allowed to wear their cleats. The mound was engineered to meet major league specifications. Ball velocity was recorded with a Trackman device (Trackman; Scottsdale, Arizona).

Each pitcher went through a pre-game warm-up period of 15 minutes consisting of dynamic warm-up and throwing to $36 \mathrm{~m}$. To best mimic training and competition pitching routines, dynamic warm-up and throwing were not regulated beyond warm-up time and the distance thrown. Following the warm-up period, players pitched four fastballs, four breaking balls, and four change-ups to a catcher receiving throws at a regulation distance $(18.4 \mathrm{~m})$. Some players elected not to throw breaking balls or change-ups if they were not comfortable with that pitch type. Three pitches of each pitch type were analyzed. Data were processed, and variables were calculated with Visual3D (C-Motion, Inc; Germantown, Maryland). Pitching models were defined using the PitchTrak model and segment coordinate systems were defined according to International Society of Biomechanics recommendations. ${ }^{23,24}$ Kinematics and kinetics were calculated from the entire pitching cycle and analyzed throughout the cycle and at key time points (high knee, front foot contact, ball release, follow-through). For the upper body segments, a top-down (distal-to-proximal) inverse dynamics approach was used for calculations. ${ }^{25}$ Shoulder distraction force is the component along the long axis of the segment coordinate system. Elbow valgus torque is the moment about the anterior/posterior axis of the segment. Variables extracted from the pitching reports included kinematics (pitching velocity, peak pelvis rotation velocity, peak trunk rotation velocity, time of peak pelvis rotation velocity, time of peak trunk rotation velocity, trunk flexion, lateral trunk tilt, hip shoulder separation at front foot contact) and kinetics (maximum elbow valgus torque, maximum shoulder distraction force, and maximum GRF).

\section{STATISTICAL ANALYSES}

Means (standard deviations (SD)) and medians (interquartile ranges (IQR)) were calculated for both descriptive statistics (i.e., age, height, and mass) and biomechanical (kinematic and kinetic) variables. Each pitch type (fastball, breaking ball, and change-up) was analyzed separately to compare those that identified as pitchers and non-pitchers. Further, each pitch type was assessed within each group. A series of t-tests and Mann-Whitney U tests were conducted to investigate the variability in pitch kinematics and kinetics between pitchers and non-pitchers. To reduce the chance of Type 1 error, a Bonferroni correction was performed, with an alpha of 0.0009 calculated. A series of analysis of variance (ANOVA) and Kruskal-Wallis tests were performed to investigate the variability of different pitch types within each group. If significant differences were observed between pitches, Tukey's or Dunn's post hoc analyses were performed for specific pitch types differences. If findings were statistically significant, Cohen's $d$ effect sizes (ES) with 95\% confidence intervals (95\% CIs) were calculated. Effect sizes were rated as small $(d=0.20-0.50)$, moderate $(d=0.50-0.80)$, and large $(d>0.80){ }^{26}$ All analyses were performed in $\mathrm{R}$ version 3.5.1 (R Core Team [2013]. R: A language and environment for statistical computing. $\mathrm{R}$ Foundation for Statistical Computing, Vienna, Austria. URL http://www.R-project.org/)

\section{RESULTS}

Data from a total of 60 adolescent baseball players 
(pitchers: $\mathrm{n}=40$; non-pitchers: $\mathrm{n}=20$ ) were analyzed (Table $1)$. There were no statistical differences in age $(p=.479)$, height $(p=.048)$, or weight $(p=.903)$. A total of 495 pitches were thrown, with pitchers throwing 342 pitches and nonpitchers throwing 153 pitches. The overall average mean pitch velocity was $29.5(3.3) \mathrm{m} / \mathrm{s}$; the mean fastball pitching velocity was $31.9(2.8) \mathrm{m} / \mathrm{s}$; the mean breaking ball pitching velocity was $27.2(2.7) \mathrm{m} / \mathrm{s}$; and the mean change-up pitching velocity was $29.0(2.4) \mathrm{m} / \mathrm{s}$. There was a moderate difference in fastball pitch velocity in favor of pitchers (pitchers: $32.6(2.6) \mathrm{m} / \mathrm{s}$, non-pitchers: 30.6 (2.7) $\mathrm{m} / \mathrm{s} ; p<.0001$, $d=0.76$ (95\% CI: $0.44,1.08)$; breaking ball pitch velocity (pitchers: $27.8(2.4) \mathrm{m} / \mathrm{s}$, non-pitchers: 25.9 (2.7) m/s; $p<$ $.0001, d=0.76$ (95\% CI: 0.40, 1.12); and a large difference for change-up pitch velocity (pitchers: $29.6(2.1) \mathrm{m} / \mathrm{s}$, nonpitchers: $27.6(2.5) \mathrm{m} / \mathrm{s} ; p<.0001, d=0.86$ (95\% CI: 0.53 , 1.24).

There was a moderate effect in favor of pitchers for greater trunk rotation velocity for fastballs (pitchers: 1053 (87) deg/s, non-pitchers: 988 (103) deg/s; $p<.0001, d=0.71$ (95\% CI: 0.39, 1.30) and change-ups (pitchers: 999 (104) $\mathrm{deg} / \mathrm{s}$, non-pitchers: 923 (107) deg/s; $p<.0001, d=0.73$ $(0.38,1.08)$. No other kinematic or kinetic variables were different for any pitches between pitchers and non-pitchers (Table 2).

Pitching velocity was faster in pitchers among all three different pitch types. Pitchers threw fastballs at greater velocity than breaking balls ( $p<.0001, d=1.92$ (95\% CI: 1.76 , 2.08)); change-ups faster than breaking balls $(p<0.0001, d=$ 0.89 (95\% CI: $0.63,1.15)$ ); and fastballs faster than changeups ( $p<.0001, d=1.28$ (95\% CI: 0.86, 1.69)). Pitchers displayed moderately greater trunk rotation velocity for fastballs compared to breaking balls $(p<.0001, d=0.59$ (95\% CI: $0.46,0.72)$ ), as well as fastballs compared to change-ups $(p<.0001, d=0.57$ (95\% CI: 0.22, 0.92)). Pitchers had moderately greater GRF when pitching fastballs in comparison to change-ups ( $p<.0001, d=0.48$ (95\% CI: $0.01,0.94)$ ). No other kinematic or kinetic variables were different between pitcher pitch types.

Non-pitchers threw fastballs faster than breaking balls ( $p$ $<.0001, d=1.74$ (95\% CI: 1.66, 1.96)) and fastballs faster than change-ups ( $p<.0001, d=1.15$ (95\% CI: $1.00,1.30)$ ). There was no difference in pitch velocity between breaking balls and change-ups ( $p=.005)$. There were no other kinematic or kinetic differences between non-pitcher pitch types.

\section{DISCUSSION}

Prior to this study, pitching biomechanics between adolescent baseball players that identify as pitchers or non-pitchers was not well-understood; such information may help inform injury risk reduction and training strategies. Therefore, the purpose of this study was to 1) analyze pitching biomechanical differences for fastball, change-ups, and curveballs between youth baseball players that identify as pitchers and non-pitchers; and 2) analyze pitching biomechanical differences between fastballs, change-ups, and curveballs within youth baseball players that identify as pitcher or non-pitchers. For all pitch types, pitchers threw an average of $2 \mathrm{~m} / \mathrm{s}$ faster than non-pitchers. Pitchers dis- played greater trunk rotation velocity for fastballs and change-ups in comparison to non-pitchers. Pitchers demonstrated greater maximum GRF for fastballs in comparison to change-ups, while non-pitchers did not demonstrate differences for maximum GRF between pitch types. All other kinematic and kinetic variables were similar between pitchers and non-pitchers for all pitch types, potentially demonstrating that pitchers utilize different trunk kinematics and GRF strategies but similar arm kinematic and kinetics in comparison to non-pitchers.

Adolescent pitchers demonstrated increased trunk rotation velocity for fastballs and change-ups in comparison to non-pitchers; further, pitchers displayed greater trunk rotation velocity for fastballs compared to both breaking balls and change-ups, while non-pitchers had similar trunk rotation velocity between all three pitch types. In this study, fastballs were delivered at a faster velocity than change-ups for both pitchers and non-pitchers. However, the discrepancies in trunk rotation velocity between groups demonstrate that pitchers potentially utilize different force production strategies for different pitch velocities. These dissimilarities in trunk rotation velocity between fastballs and changeups are similar to findings in a previous study using trunkbased triaxial global positioning monitoring systems in collegiate baseball pitchers. ${ }^{27}$ The trunk has been observed to produce up to $50 \%$ of kinetic energy during the pitching motion. ${ }^{28}$ Thus, faster trunk rotation velocity warrants greater force transfer through the upper extremity and increasing ball propulsion. ${ }^{29,30}$ Also, greater trunk rotation strategies can increase forces through the upper extremity, ${ }^{31}$ while ineffective timing between pelvis and trunk rotation can inhibit ball propulsion velocity. $11,23,29$

Baseball pitchers may use multiple strategies for force generation in comparison to non-pitchers between different pitch types. Non-pitchers may use one force strategy for all pitch types and reduce pitch velocity by absorbing increased forces through the upper extremity instead of using this force for ball propulsion. The results of this study support this fact as non-pitchers threw with $2 \mathrm{~m} / \mathrm{s}$ (5 mph) less velocity but demonstrated no significant difference in shoulder or elbow kinetics. There were no other differences in kinematics or kinetics between adolescent pitchers and non-pitchers. Coaches need to consider the potential for increased upper extremity forces when pitching different pitch types while monitoring non-pitcher's loading strategies.

A positive linear relationship has been previously observed between pitching velocity and elbow valgus torque in youth pitchers. ${ }^{32,33}$ Pitching places high stress on the musculoskeletal system. Pitchers that produce more force toward ball propulsion instead of upper extremity joint forces are potentially reducing injury risk. ${ }^{11,15}$ Compared to adolescent baseball players that primarily identify as pitchers, players that identify as non-pitchers may potentially utilize similar upper extremity pitching mechanics that generate decreased velocity. Coaches can use this information when considering pitch count and loading progressions for nonpitchers. For example, baseball players that primarily play a fielding position with intermittent pitching should be given more recovery consideration than a primary pitcher for an equal number of pitches. 
Table 2: Kinematic and Kinetic Comparison of Pitchers and Non-Pitchers

\begin{tabular}{|c|c|c|c|c|c|c|c|c|c|}
\hline & \multicolumn{3}{|c|}{ Fastball } & \multicolumn{3}{|c|}{ Curveball } & \multicolumn{3}{|c|}{ Change-up } \\
\hline & Pitchers & $\begin{array}{c}\text { Non- } \\
\text { Pitchers }\end{array}$ & $\begin{array}{c}\text { p-value/ } \\
\text { ES(Cl) }\end{array}$ & Pitchers & $\begin{array}{c}\text { Non- } \\
\text { Pitchers }\end{array}$ & $\begin{array}{c}\text { p-value/ } \\
\text { ES(Cl) }\end{array}$ & Pitchers & $\begin{array}{c}\text { Non- } \\
\text { Pitchers }\end{array}$ & $\begin{array}{c}\text { p-value/ } \\
\text { ES(Cl) }\end{array}$ \\
\hline Pelvis Rotation Velocity (deg/s) & $\begin{array}{c}670 \\
(109)\end{array}$ & $\begin{array}{l}657 \\
(96)\end{array}$ & .398 & $\begin{array}{c}634 \\
(100)\end{array}$ & $\begin{array}{c}619 \\
(105)\end{array}$ & .424 & $\begin{array}{l}624 \\
(87)\end{array}$ & $\begin{array}{l}599 \\
(99)\end{array}$ & .122 \\
\hline Trunk Rotation Velocity (deg/s) & $\begin{array}{l}1053 \\
(87)\end{array}$ & $\begin{array}{c}988 \\
(103)\end{array}$ & $\begin{array}{c}<.0001^{*} \\
0.71 \\
(0.39,1.30)\end{array}$ & $\begin{array}{l}1000 \\
(93)\end{array}$ & $\begin{array}{c}941 \\
(125)\end{array}$ & .005 & $\begin{array}{c}999 \\
(104)\end{array}$ & $\begin{array}{c}923 \\
(107)\end{array}$ & $\begin{array}{c}<.0001^{*} \\
0.73 \\
(0.38,1.08)\end{array}$ \\
\hline Time to peak pelvis rotation velocity (s) & $\begin{array}{c}810 \\
(741-879)\end{array}$ & $\begin{array}{c}784 \\
(708-860)\end{array}$ & .085 & $\begin{array}{c}792 \\
(706-878)\end{array}$ & $\begin{array}{c}771 \\
(684-860)\end{array}$ & .545 & $\begin{array}{c}813 \\
(724-904)\end{array}$ & $\begin{array}{c}780 \\
(696-864)\end{array}$ & .336 \\
\hline Time to peak trunk rotation velocity $(\mathrm{s})$ & $\begin{array}{c}832 \\
(769-896)\end{array}$ & $\begin{array}{c}812 \\
(742-882)\end{array}$ & .089 & $\begin{array}{c}806 \\
(716-896)\end{array}$ & $\begin{array}{c}808 \\
(744-872)\end{array}$ & .485 & $\begin{array}{c}840 \\
(750-930)\end{array}$ & $\begin{array}{c}820 \\
(750-890)\end{array}$ & .333 \\
\hline Trunk flexion (deg) & $\begin{array}{c}23.4 \\
(18.3-27.4) \\
\end{array}$ & $\begin{array}{c}20.9 \\
(17.1-23.8) \\
\end{array}$ & .003 & $\begin{array}{c}22.2 \\
(17.6-26.8) \\
\end{array}$ & $\begin{array}{c}18.9 \\
(13.0-24.8) \\
\end{array}$ & .004 & $\begin{array}{c}22.5 \\
(18.7-27.3) \\
\end{array}$ & $\begin{array}{c}20.4 \\
(14.3-26.5) \\
\end{array}$ & .008 \\
\hline Lateral Trunk Tilt (deg) & $\begin{array}{c}10.1 \\
(2.3-17.9) \\
\end{array}$ & $\begin{array}{c}6.9 \\
(1.9-12.0) \\
\end{array}$ & .252 & $\begin{array}{c}9.0 \\
(0-13.8) \\
\end{array}$ & $\begin{array}{c}4.2 \\
(0-9.9)\end{array}$ & .044 & $\begin{array}{c}5.5 \\
(0-13.9) \\
\end{array}$ & $\begin{array}{c}4.4 \\
(0-8.9) \\
\end{array}$ & .131 \\
\hline Hip Shoulder Separation (deg) & $\begin{array}{c}49.1 \\
(41.4-56.7)\end{array}$ & $\begin{array}{c}43.5 \\
(35.0-52.1)\end{array}$ & .084 & $\begin{array}{c}49.1 \\
(37.8-56.0)\end{array}$ & $\begin{array}{c}38.0 \\
(30.1-45.9)\end{array}$ & .023 & $\begin{array}{c}44.2 \\
(36.3-52.1)\end{array}$ & $\begin{array}{c}38.8 \\
(30.6-46.9)\end{array}$ & .068 \\
\hline $\begin{array}{l}\text { Maximum Elbow Valgus Torque } \\
\text { (\%BWxH) }\end{array}$ & $\begin{array}{c}3.1 \\
(2.6-3.6)\end{array}$ & $\begin{array}{c}2.8 \\
(2.2-3.4)\end{array}$ & .365 & $\begin{array}{c}2.9 \\
(2.3-3.3)\end{array}$ & $\begin{array}{c}2.8 \\
(2.3-3.3)\end{array}$ & .233 & $\begin{array}{c}2.7 \\
(2.2-3.2)\end{array}$ & $\begin{array}{c}2.5 \\
(2.3-2.9)\end{array}$ & .042 \\
\hline $\begin{array}{c}\text { Maximum Shoulder Distraction Force } \\
(\% \mathrm{BW})\end{array}$ & $\begin{array}{c}120 \\
(104-136) \\
\end{array}$ & $\begin{array}{c}105 \\
(90-120) \\
\end{array}$ & .013 & $\begin{array}{c}113 \\
(95-130) \\
\end{array}$ & $\begin{array}{c}94 \\
(84-104) \\
\end{array}$ & .120 & $\begin{array}{c}106 \\
(94-117) \\
\end{array}$ & $\begin{array}{c}93 \\
(76-110)\end{array}$ & .039 \\
\hline $\begin{array}{c}\text { Maximum Ground Reaction Force } \\
\text { (\%BW) }\end{array}$ & $\begin{array}{l}217 \\
(47)\end{array}$ & $\begin{array}{l}193 \\
(45)\end{array}$ & .002 & $\begin{array}{l}190 \\
(39)\end{array}$ & $\begin{array}{l}187 \\
(43)\end{array}$ & .131 & $\begin{array}{l}197 \\
(37)\end{array}$ & $\begin{array}{l}181 \\
(40)\end{array}$ & .027 \\
\hline
\end{tabular}

Means and standard deviation are reported as mean (SD), medians and interquartile range are reported as median (IQR); ES noted with $95 \% \mathrm{CI}$ $\mathrm{BW}=$ Body Weight, $\mathrm{H}=$ Height

"After Bonferroni correction, significance was set at $p<0.0009$ 
Adolescent pitchers demonstrated greater maximum GRF pitching fastballs compared to change-ups, while nonpitchers had similar maximum GRF between all pitch types. Maximum GRF has a direct link to pitch velocity. ${ }^{34}$ During the pitching motion, the lower extremity serves as a counterforce to slow the lower extremity in order to create the trunk and upper extremity acceleration. ${ }^{30}$ Greater GRF allows for increased momentum and potential pitch velocity generation. ${ }^{35}$ However, non-pitchers did not demonstrate differences in maximum GRF between pitch types. One possible explanation is that pitchers produce improved resistance to knee flexion while producing an extension knee moment from front contact to release. ${ }^{36,37}$ Maximum GRF is produced during the late arm cocking phase. ${ }^{34,35}$ The ability of pitchers to resist knee flexion as lower extremity forces transition from a posterior to an anterior position during late cocking may allow for improved force transfer to the trunk and to ball propulsion. Non-pitchers may utilize increased knee flexion during the pitching motion instead of producing different forces to generate different pitch velocities for different pitch types. These discrepancies in lower extremity transfer may lead to increased injury risk; however, further research is required to understand the relationship between GRF, knee flexion, and injuries within baseball pitchers.

Currently, biomechanical research on pitching has focused on baseball players that are primarily pitchers. ${ }^{15}$ However, at the adolescent level, youth and amateur competitive baseball athletes may participate in multiple positions, including pitching. Future research is required to understand the relationship between pitching as a primary or secondary position and its association with injury risk and optimal loading strategies.

\section{STRENGTHS AND LIMITATIONS}

There were several strengths and limitations in this study related to design and statistical analysis. This study utilized a standardized pitching biomechanical model with international guidelines, ${ }^{23,24}$ increasing the repeatability of this study. Reflective markers were placed directly on bony landmarks to minimize error due to skin movement between the markers and the anatomical landmarks being signified.

Incorporating adolescent baseball players that identify as primarily pitchers and non-pitchers increases the generalizability of these findings to all baseball players. While baseball players were constrained to a specific warm-up time, the exact warm-up exercises were personalized to replicate individual practice and game scenarios, which may reduce the repeatability of this investigation. These participants may be developing athletes, which may limit the external validity of these findings to other higher-level baseball populations. Further, skill level was not stratified in these analyses, potentially confounding these results

A large cohort of 60 baseball players participated. Each pitch type was pitched in a specific order, which potentially could create an order effect. Not all baseball players threw each pitch type; while the authors attempted to control for this in the statistical methodology, this decreases the robustness of the data. Due to the multiple statistical analyses, a Bonferroni correction was utilized to decrease the probability of type 1 error. The low p-value threshold could limit the identification of important differences between groups. However, to assist in counteracting this possibility, effect size indices were calculated to note the overall strength of the effect.

\section{CONCLUSION}

Adolescent baseball players that identified primarily as pitchers had overall similar kinematics and kinetics in comparison to players that primarily identified as non-pitchers despite throwing at greater velocity for all pitch types. Adolescent pitchers produced greater trunk rotation velocity for fastballs in comparison to change-ups, while non-pitchers produced similar trunk rotation velocity between pitch types. Further, pitchers demonstrated decreased maximum GRF during change-ups in comparison to fastballs, while non-pitchers produced similar GRF between all pitch types. These data suggest that adolescent baseball players who identify primarily as pitchers may use favorable trunk and ground reaction force strategies for ball propulsion, but use similar upper extremity kinematics during pitching.

\section{CONFLICTS OF INTEREST}

No authors report any conflicts of interest.

Submitted: April 27, 2020 CDT, Accepted: October 10, 2020 CDT 


\section{REFERENCES}

1. Kerr ZY, Roos KG, Djoko A, Dompier TP, Marshall SW. Rankings of high school sports injury rates differ based on time loss assessments. Clin J Sport Med. 2016;27(6):548-551.

2. Pytiak AV, Kraeutler MJ, Currie DW, McCarty EC, Comstock RD. An epidemiological comparison of elbow injuries among United States high school baseball and softball players, 2005-2006 through 2014-2015. Sports Health. 2018;10(2):119-124. doi:1 $\underline{0.1177 / 1941738117736493}$

3. Saper MG, Pierpoint LA, Liu W, Comstock RD, Polousky JD, Andrews JR. Epidemiology of shoulder and elbow injuries among United States high school baseball players: school years 2005-2006 through 2014-2015. Am J Sports Med. 2018;46(1):37-43. doi:1 $\underline{0.1177 / 0363546517734172}$

4. Shanley E, Rauh MJ, Michener LA, Ellenbecker TS. Incidence of injuries in high school softball and baseball players. J Athl Train. 2011;46(6):648-654.

5. Statista. Number of participants in baseball in the United States from 2006 to 2017 (in millions). http s://www.statista.com/statistics/191626/participants-i n-baseball-in-the-us-since-2006/. Published 2018. Accessed March 18, 2019.

6. Little League Baseball. https://www.littleleague.or g/. Published 2018. Accessed March 18, 2019.

7. National Federation of State High School Associations. High School Athletics Participation Results. https://www.nfhs.org/ParticipationStatistics/ ParticipationStatistics/. Published 2018. Accessed March 18, 2019.

8. Lyman S, Fleisig GS, Andrews JR, Osinski ED. Effect of pitch type, pitch count, and pitching mechanics on risk of elbow and shoulder pain in youth baseball pitchers. Am J Sports Med. 2002;30(4):463-468.

9. Lyman S, Fleisig GS. Baseball injuries. In: Epidemiology of Pediatric Sports Injuries. Vol 49. Karger Publishers; 2005:9-30.

10. Fleisig GS, Andrews JR, Cutter GR, et al. Risk of serious injury for young baseball pitchers: a 10-year prospective study. Am J Sports med. 2011;39(2):253-257.

11. Fleisig GS, Barrentine SW, Escamilla RF, Andrews JR. Biomechanics of overhand throwing with implications for injuries. Sports Med. 1996;21(6):421-437.
12. Fleisig GS, Andrews JR, Dillman CJ, Escamilla RF. Kinetics of baseball pitching with implications about injury mechanisms. Am J Sports Med.

1995;23(2):233-239.

13. Davis J, Limpisvasti O, Fluhme D, et al. The effect of pitching biomechanics on the upper extremity in youth and adolescent baseball pitchers. Am J Sports Med. 2009;37(8):1484-1491.

14. Douoguih WA, Dolce DL, Lincoln AE. Early cocking phase mechanics and upper extremity surgery risk in starting professional baseball pitchers. Orthop J Sports Med. 2015;3(4):2325967115581594. do i:10.1177/2325967115581594

15. Fleisig GS, Barrentine SW, Zheng N, Escamilla RF, Andrews JR. Kinematic and kinetic comparison of baseball pitching among various levels of development. J Biomech. 1999;32(12):1371-1375.

16. Fleisig GS, Laughlin WA, Aune KT, Cain EL, Dugas JR, Andrews JR. Differences among fastball, curveball, and change-up pitching biomechanics across various levels of baseball. Sports Biomech. 2016;15(2):128-138.

17. Dun S, Loftice J, Fleisig GS, Kingsley D, Andrews JR. A biomechanical comparison of youth baseball pitches: is the curveball potentially harmful? Am J Sports Med. 2008;36(4):686-692.

18. Nissen CW, Westwell M, Õunpuu S, Patel M, Solomito M, Tate J. A biomechanical comparison of the fastball and curveball in adolescent baseball pitchers. Am J Sports Med. 2009;37(8):1492-1498.

19. Fleisig GS, Kingsley DS, Loftice JW, et al. Kinetic comparison among the fastball, curveball, change-up, and slider in collegiate baseball pitchers. Am J Sports Med. 2006;34(3):423-430.

20. Barrentine SW, Matsuo T, Escamilla RF, Fleisig GS, Andrews JR. Kinematic analysis of the wrist and forearm during baseball pitching. J Appl Biomech. 1998;14(1):24-39.

21. Solomito MJ, Garibay EJ, Woods JR, Õunpuu S, Nissen CW. Evaluation of wrist and forearm motion in college-aged baseball pitchers. Sports Biomech. 2014;13(4):320-331.

22. Keller RA, Marshall NE, Guest J-M, Okoroha KR, Jung EK, Moutzouros V. Major League Baseball pitch velocity and pitch type associated with risk of ulnar collateral ligament injury. J Shoulder Elbow Surg. 2016;25(4):671-675. 
23. Aguinaldo AL, Buttermore J, Chambers H. Effects of upper trunk rotation on shoulder joint torque among baseball pitchers of various levels. J Appl Biomech. 2007;23(1):42-51.

24. Wu G, Van der Helm FC, Veeger HD, et al. ISB recommendation on definitions of joint coordinate systems of various joints for the reporting of human joint motion-Part II: shoulder, elbow, wrist and hand. J Biomech. 2005;38(5):981-992.

25. Feltner ME, Dapena J. Three-dimensional interactions in a two-segment kinetic chain. Part I: General model. J Appl Biomech. 1989;5(4):403-419.

26. Cohen J. Statistical Power Analysis for the Behavioral Sciences. Academic Press; 2013.

27. Bullock GS, Schmitt AC, Chasse P, Little BA, Diehl LH, Butler RJ. Differences in PlayerLoad and pitch type in collegiate baseball players. Sports Biomech. 2019:1-9.

28. Toyoshima S, Hoshikawa T, Miyashita M, Oguri T. Contribution of the body parts to throwing performance. In: Biomechanics IV. Springer; 1974:169-174.

29. Oyama S, Yu B, Blackburn JT, Padua DA, Li L, Myers JB. Improper trunk rotation sequence is associated with increased maximal shoulder external rotation angle and shoulder joint force in high school baseball pitchers. Am J Sports Med.

2014;42(9):2089-2094.

30. Putnam CA. Sequential motions of body segments in striking and throwing skills: descriptions and explanations. J Biomech. 1993;26:125-135.
31. Cohen AD, Garibay EJ, Solomito MJ. The association among trunk rotation, ball velocity, and the elbow varus moment in collegiate-level baseball pitchers. Am J Sports Med. 2019;47(12):2816-2820.

32. Hurd WJ, Jazayeri R, Mohr K, Limpisvasti O, ElAttrache NS, Kaufman KR. Pitch velocity is a predictor of medial elbow distraction forces in the uninjured high school-aged baseball pitcher. Sports Health. 2012;4(5):415-418.

33. Luera MJ, Dowling B, Magrini MA, Muddle TW, Colquhoun RJ, Jenkins ND. Role of rotational kinematics in minimizing elbow varus torques for professional versus high school pitchers. Orthop J Sports Med. 2018;6(3):2325967118760780.

34. McNally MP, Borstad JD, Oñate JA, Chaudhari AM. Stride leg ground reaction forces predict throwing velocity in adult recreational baseball pitchers. $J$ Strength Cond Res. 2015;29(10):2708-2715.

35. MacWilliams BA, Choi T, Perezous MK, Chao EY, McFarland EG. Characteristic ground-reaction forces in baseball pitching. Am J Sports Med. 1998;26(1):66-71.

36. Matsuo T, Escamilla RF, Fleisig GS, Barrentine SW, Andrews JR. Comparison of kinematic and temporal parameters between different pitch velocity groups. J Appl Biomech. 2001;17(1):1-13.

37. Whiting WC, Gregor RJ, Halushka M. Body segment and release parameter contributions to newrules javelin throwing. J Appl Biomech. 1991;7(2):111-124. 